\title{
Investigation of $\beta-\mathrm{Ga}_{2} \mathrm{O}_{3}$-based HEMT for Low Noise Amplification and RF Application
}

\author{
R. Singh ${ }^{1}$, T. R. Lenka ${ }^{1}$, D. K. Panda ${ }^{2}$, and H. P.T. Nguyen ${ }^{3}$
}

\begin{abstract}
Here we demonstrate a two-dimensional $\beta$ gallium oxide-based high electron mobility transistor (HEMT) comprising of a finite gap-access region gap $\left(\boldsymbol{L}_{A R G}\right)$ in Ohmiccontact access regions with record transconductance linearity. Apart from limiting two-dimensional electron gas (2DEG) density $\boldsymbol{n}_{s}$ dependency on gate voltage, higher saturation current is estimated for the proposed design. Since the access regions length directly affects the Capacitance of the device and resultant switching applications. In this work, the effect of the gate-source and gate-drain length on device linearity is performed using Atlas-2D simulations. $\boldsymbol{C}-\boldsymbol{V}$ characteristics of the proposed device are explained based on the physical explanation and validated using appropriate models. The higher values of transconductance $\mathbf{g}_{m}$ and current gain cut-off frequency $\boldsymbol{f}_{T}$ on a large span of operating voltages ensure improved transistor performance for low-noise amplification and $\mathrm{RF}$ application and are reported for the first time.
\end{abstract}

Index Terms-2DEG, $\mathrm{AIN} / \mathrm{\beta}-\mathrm{Ga}_{2} \mathrm{O}_{3}, \quad$ access-regions, Capacitance, $\boldsymbol{C}-\boldsymbol{V}$ characteristics, Gaussian-doping, HEMT, linearity, quasi-saturation, source-resistance, transconductance.

\section{INTRODUCTION}

$\mathrm{H}$ igh-electron-mobility-transistors have shown outperformance in terms of high-frequency and highvoltage operations. Higher two-dimensional-electron gas (2DEG) density $n_{s} \sim 10^{12}-10^{13} \mathrm{~cm}^{-3}$ and 2DEG mobility of $1200-1500 \mathrm{~cm}^{2} \mathrm{~V}^{-1} \mathrm{~s}^{-1}$ make GaN-based HEMTs the preferable choice for high-frequency electronics applications. However currently, a relatively new semiconductor material galliumoxide $\left(\mathrm{Ga}_{2} \mathrm{O}_{3}\right)$ is being explored at a fast pace for potential highpower and high-frequency electronics emerging applications. Out of its five phases, the $\beta$-phase is found to be the most stable and has some interesting properties like large bandgap $E_{\mathrm{g}} \sim 4.5$ $4.9 \mathrm{eV}$, critical electrical field $E_{\mathrm{C}} \sim 8-9 \mathrm{MV} / \mathrm{cm}$, and availability of economical single crystal substrates grown using melt-based growth techniques [1]-[4]. Despite the low electron mobility $\mu_{n}$ $\sim 150-200 \mathrm{~cm}^{2} \mathrm{~V}^{-1} \mathrm{~s}^{-1}$ issue, researchers have shown enough pieces of evidence that $\mathrm{Ga}_{2} \mathrm{O}_{3}$ can complement existing $\mathrm{GaN}$ and $\mathrm{SiC}$ technology [5]-[7].

Despite high carrier density and carrier velocity, HEMTs generally suffer from nonlinearity issues. The gain i.e.

${ }^{1}$ R. Singh and T. R. Lenka are with the Microelectronics and VLSI Design Group, Department of Electronics \& Communication Engineering, National Institute of Technology Silchar, Assam, 788010, India. (rajan.1114@gmail.com ; trlenka@ieee.org)

${ }^{2}$ D. K. Panda is with the Microelectronics and VLSI Design Group, School of Electronics, VIT-AP University, Amaravati, AP, 522237 India. (deepakiitkgp04@gmail.com) transconductance $g_{m}$, and current-gain cut-off frequency $f_{T}$, after reaching their peak values, show a rapid fall with gate bias [8], [9]. This bias-dependent nonlinearity affects the noise performance of amplifiers and is attributed to the high resistivity of Ohmic-contact access regions. Several methods have been proposed so far to ease this effect using high-doping in the source-access region at the heterointerface [8], and multichannel structures [10], [11] and achieved good linearity by reducing access-region resistance. Earlier this was explained based on enhanced electron-phonon interactions in $\mathrm{GaN}$ HEMTs [9]. Lately, several three-dimensional (3D) structures are proposed comprising $3 \mathrm{DEG}[12]$, and lateral-gated $3 \mathrm{D}$ structures like junction field-effect transistors (JFETs) with lateral depletion [13]. Few of them have lower breakdown voltage while most have sophisticated designs.

Access region resistance has been identified as the main source of nonlinearity in high-electron-mobility transistors (HEMTs), and surface potential based analytical model and its temperature dependence for GaN based HEMT has been reported [14], and experimental analysis of source resistance in InGaAs/InAlAs HEMTs [15]. Furthermore, parasitic source resistance was measured based on varying gate bias and gate length of GaN based heterostructure FETs (HFETs) [16], and optimization of nonlinear access resistance in $\beta-\mathrm{Ga}_{2} \mathrm{O}_{3}$ based HEMTs has been reported [17]. These various methods envisaged to minimize dynamic access resistance broadly ranges from high-doping in the access region and threedimensional structure comprising three-dimensional electron gas (3DEG) and lateral depletion. However, the effects of a particular method proposed for reducing access resistance on the capacitances and resulting linearity of the device require more investigation.

In this paper, TCAD simulations have been performed, on a $\beta$ - $\mathrm{Ga}_{2} \mathrm{O}_{3}$ HEMT with access-region-gaps, to quantify the effects of varying access-region lengths on the $C-V$ behavior and linearity of the device. The $C-V$ behavior is validated by appropriate analytical models reported earlier. The Section II explains device structure and simulation outline followed by results and discussion in section III. The paper is concluded in Section IV.

${ }^{3}$ H. P. T. Nguyen is with the Department of Electrical and Computer Engineering, New Jersey Institute of Technology, Newark, New Jersey, 07102, USA. (hieu.p.nguyen@niit.edu ) 


\section{DEVICE DESIGN AND SIMULATIONS FRAMEWORK}

The schematic of the device under test is shown in Fig. 1 (a). The epitaxial layer sequence consists of 50-nm width $\beta-\mathrm{Ga}_{2} \mathrm{O}_{3}$ buffer layer on a semi-insulating $\beta-\mathrm{Ga}_{2} \mathrm{O}_{3}$ substrate, followed by $10-\mathrm{nm}$ width AlN barrier layer. The buffer layer has a doping concentration of $10^{16} \mathrm{~cm}^{-3}$, while the barrier layer is undoped. The gate contact is Schottky type, while drain and source contacts are the Ohmic types with a contact resistance of 0.4 ohm-mm as given in [18]. The device has the gate-length $L_{G}$ of $0.1 \mu \mathrm{m}$, and equal gate-source $L_{G S}$ and gate-drain length $L_{G D}$ of $0.7 \mu \mathrm{m}$. The AlN barrier is contracted laterally to create a finite gap-access region gap $L_{A R G}$ of $0.05 \mu \mathrm{m}$. This is done to minimize charge depletion in the vicinity of barrier end and source/drain regions. The same can be done easily using more popular self-aligned technology, which is leveraged here for potential gain in device linearity. The schematic of conventional HEMT, used for comparing results, is also shown in Fig. 1 (b). The device width is set as $50 \mu \mathrm{m}$ and all the reported quantities are normalized on this dimension. All simulations and numerical computations are performed using ATLAS 2D device commercial software [19], and MathWorks - MATLAB [20] respectively. For a drain bias up to $15 \mathrm{~V}$, and a critical field of $1.54 \mathrm{kV} / \mathrm{cm}[21]$ the ratio $V_{D S} / E_{C}$ is equal to $1 \mu \mathrm{m}$. Therefore to keep the maximum electric field in access regions below $E_{C}$, the overall lateral length of the device i. e. source-drain distance $L_{S D}$ is kept at $1.5 \mu \mathrm{m}$.

The $\beta-\mathrm{Ga}_{2} \mathrm{O}_{3}$ material parameters such as energy bandgap $E_{G}$, conduction and valence-band density $N_{C}$ and $N_{V}$, dielectric permittivity $\varepsilon_{r}$ among others are taken from [4], [22]. The duo polarization models - spontaneous and piezoelectric are enabled for AlN material as provided in [19]. To capture the carrier velocity saturation effect in the $\beta-\mathrm{Ga}_{2} \mathrm{O}_{3}$ channel, a fielddependent mobility model is invoked among others like Shockley-Read-Hall (SRH) recombination, and Fermi Dirac statistics for carrier concentration. The user-defined parameters are given in Table 1, while the rest all for materials and models are used as default given in [19]. To capture the velocity saturation effect in velocity-field characteristics, a negative differential mobility model as given in [19] is used and default model parameters are replaced by values reported in [21].

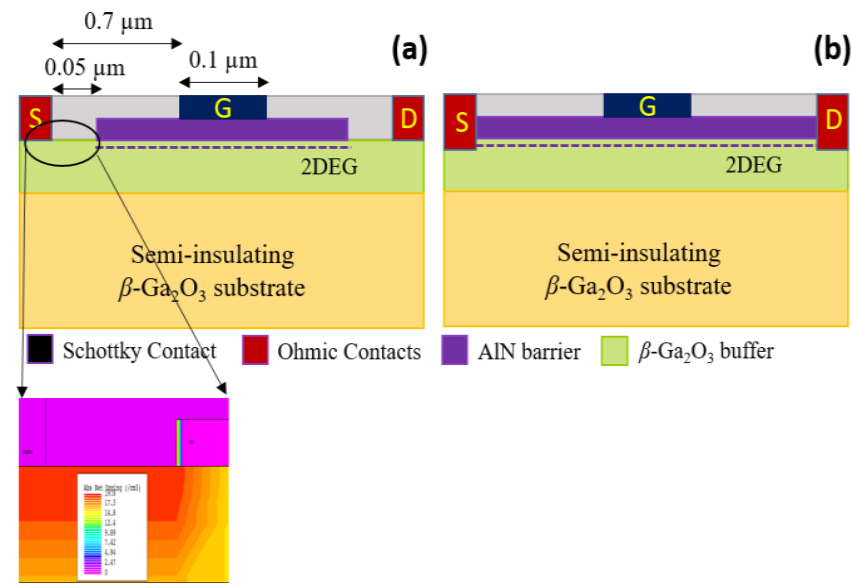

Fig. 1. Schematic view of the AIN/ $\beta-\mathrm{Ga}_{2} \mathrm{O}_{3} \mathrm{HEMT}$ device structures (a) device under test, Enlarged: Gaussian doping extending from Ohmiccontact to barrier edge (b) conventional HEMT, Ohmic contacts extended up to few $\mathrm{nm}$ in barrier layer (Dimensions not scaled).

$$
\mu_{n}(E)=\frac{\mu_{0}+\frac{v_{s a t}}{E}\left(\frac{E}{E_{C}}\right)^{\gamma}}{1+\left(\frac{E}{E_{C}}\right)^{\gamma}}
$$

Table I

$\beta$ - $\mathrm{Ga}_{2} \mathrm{O}_{3}$ material and parallel field mobility model parameters

\begin{tabular}{lll}
\hline \hline Symbol & Quantity & Value (unit) \\
\hline $\mathcal{E}_{g}$ & Energy bandgap & $4.9 \mathrm{eV}$ \\
$\mathcal{N}_{C}$ & Conduction band density & $3.6 \times 10^{18} \mathrm{~cm}^{-3}$ \\
$\mathcal{N}_{\mathcal{V}}$ & Valence band density & $2.86 \times 10^{20} \mathrm{~cm}^{-3}$ \\
$\chi$ & Electron affinity & $3.15 \mathrm{eV}$ \\
$\varepsilon_{0}$ & Static dielectric constant & 10 \\
$\mu_{0}$ & Low field mobility & $140 \mathrm{~cm}^{2} \mathrm{~V}^{-1} \mathrm{~s}^{-1}$ \\
$v_{s a t}$ & Saturation velocity & $1.5 \times 10^{7} \mathrm{~cm} \mathrm{~s}^{-1}$ \\
$\mathcal{E}_{C}$ & Critical field & $1.54 \times 10^{5}$ \\
$y$ & Constant & 2.47 \\
\hline \hline
\end{tabular}

\section{RESULTS AND DISCUSSION}

\section{A. Limit to Linearity}

Since in HEMT, 2DEG density $n_{s}$ has a profound effect on device operation and is a complex function of gate voltage. So it is vital to consider this bias-dependent behavior of 2DEG density in linearity analysis of the HEMTs. The explicit relation between $n_{s}$ and $V_{G S}$, as given in [23], can be written as follows:

$$
\begin{array}{r}
n_{s}\left(V_{G S}\right)=\frac{C_{g} V_{g_{\text {eff }} e f f}}{q} \frac{V_{t h}\left[1-\ln \left(\beta V_{g_{\text {eff }}}\right)\right]}{-\frac{\gamma_{0}}{3}\left(\frac{C_{g} V_{g_{-} e f f}}{q}\right)^{2 / 3}} \\
V_{g_{\text {eff }}\left(1+\frac{V_{\text {th }}}{V_{g_{\text {eff }}}}\right)} \\
+\frac{2}{3} \gamma_{0}\left(\frac{C_{g} V_{g_{-} e f f}}{q}\right)^{2 / 3}
\end{array}
$$

where, $C_{g}=\epsilon_{A l N} / d_{A l N}$ is the gate-capacitance, $V_{g_{-} e f f}=$ $V_{G S}-V_{O F F}$ is the effective gate voltage, $V_{t h}$ is the thermal voltage $0.0259 \mathrm{~V}$ at $300 \mathrm{~K}$. $V_{g_{-} e f f_{-} n}, V_{g_{-} e f f_{-} d}$ are functions of $V_{g_{-} e f f}$ is given as $V_{g_{-} e f f}\left(\alpha_{x} / \sqrt{V_{g_{-} e f f}^{2}+\alpha_{x}^{2}}\right)$, where $\alpha_{n}=$ $\exp (1) / \beta, \alpha_{d}=1 / \beta$, and $\beta=C_{g} / q D V_{t h}$, where $\mathrm{D}$ is the density of states. The $2 \mathrm{DEG}$ density $n_{s}$ is numerically computed using MATLAB and plotted against $V_{G S}$ for the proposed $\mathrm{AlN} / \beta-\mathrm{Ga}_{2} \mathrm{O}_{3} \mathrm{HEMT}$ as shown in Fig 2. It can be seen that $2 \mathrm{DEG}$ density rises vertically once $V_{G S}>V_{O F F}$ and gradually saturates for higher gate voltages. As $n_{s}$ increases, electron effective velocity decrease as $v_{\text {eff }} \sim 1 / \sqrt{n_{s}}$ and subsequently a similar drop in values of $\mathrm{g}_{m}$ and $f_{T}$ can be expected due to quasi-saturation of electron velocity [8], [9]. This bias-dependent effect of $n_{s}$ on $V_{G S}$ looks more evident in submicron devices as $L_{G S}$ is kept short for optimum RF performance. To circumvent this $n_{s}$ variation in the channel ends near source/drain contacts, highly doped (Gaussian-type), finite access-area gaps $L_{A R G}$ have been introduced in the proposed design. This is validated based on the variation in carrier density versus $V_{G S}$ for different $L_{A R G}$ using TCAD simulations and is shown in inset of Fig. 2. 
The results obtained are on the expected lines. The intensity of bias dependent behavior of $n_{S}$ with $\mathrm{V}_{\mathrm{GS}}$ significantly reduces and becomes constant beyond $\mathrm{V}_{\mathrm{GS}}=2 \mathrm{~V}$ for all values of $L_{A R G}$. Therefore, it can be concluded that in access regions, higher values of $L_{A R G}$ pointedly restricted percentage rise in carrier concentration with increasing gate voltages.

The $I_{D}-V_{D S}$ characteristics of the proposed as well as conventional HEMTs are shown in Fig. 3. Maximum drain current $I_{D M A X}$ of 0.32 and $0.1 \mathrm{~A} / \mathrm{mm}$ is found for the device with $L_{A R G}$ and conventional one respectively. Over $200 \%$ rise in saturation current is observed for the proposed device. This is attributed to bias-independent high doping in source/drain access regions. Moreover, lower values of ON-resistance $R_{O N}$ corresponding to the proposed device over conventional structure ensure lower net conduction losses. Furthermore, the lower knee voltage provides a large output voltage swing.

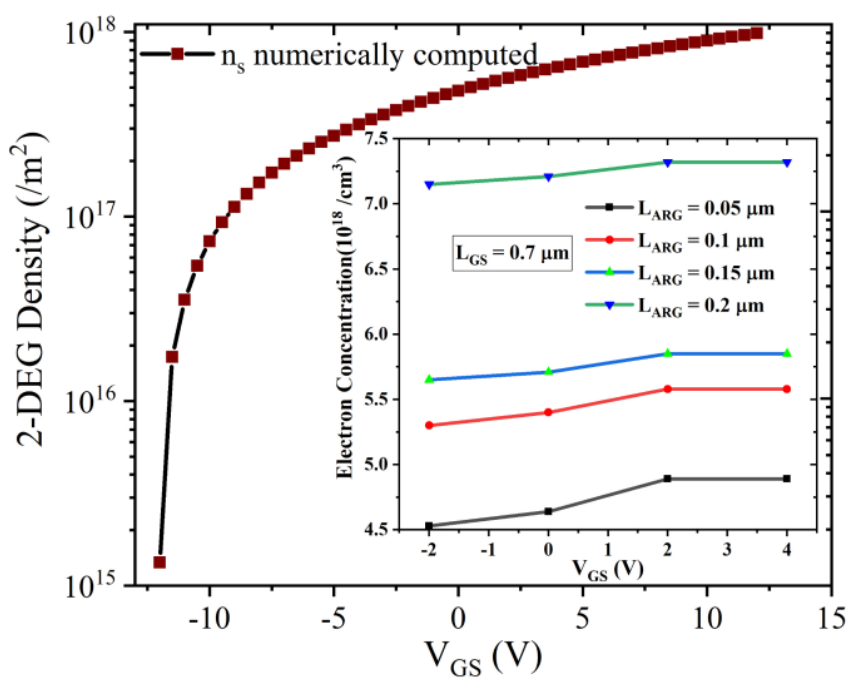

Fig. 2. Variation of 2DEG density $n_{s}$ versus gate-voltage $V_{G s}$ for proposed HEMT design. Inset: Effect of change in $L_{A R G}$ on electron concentration in source access region. The rate of increase in $n_{s}$ comes down with increase in $L_{A R G}$.

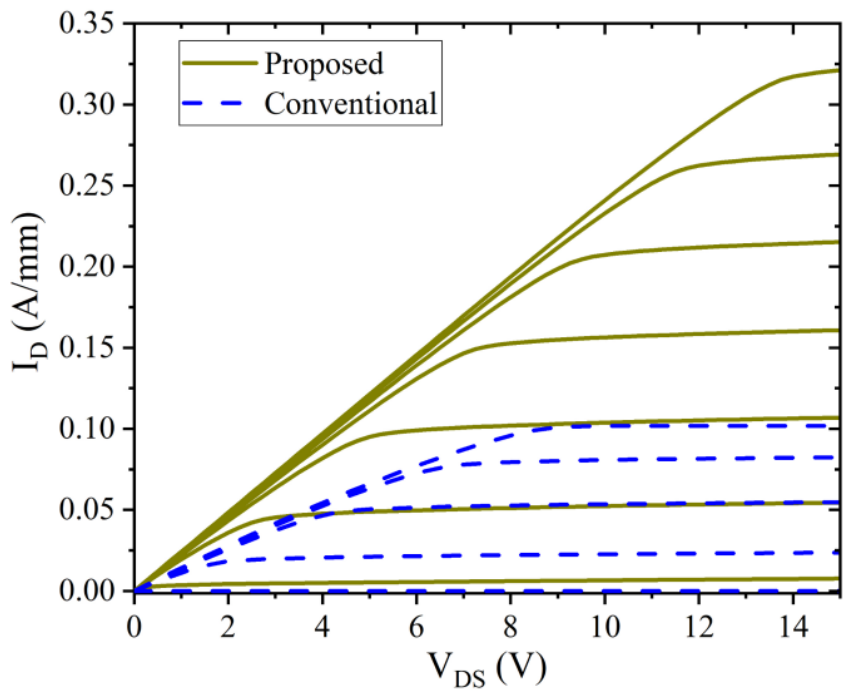

Fig. 3. $I_{D}-V_{D S}$ characteristics of the proposed and conventional HEMT designs, $\mathrm{V}_{\mathrm{GS}}$ is varied from -12 to $0 \mathrm{~V}$ and -8 to $0 \mathrm{~V}$ in step of $2 \mathrm{~V}$.

\section{B. Effect of Access Region Length}

As the access region length changes, the overall access resistance also changes. To evaluate the effect of the access region lengths on the linearity of the device, two different cases have been considered. In the first set of simulations, $\mathrm{L}_{\mathrm{GS}}$ decreases, and $\mathrm{L}_{\mathrm{GD}}$ increases in the step of $0.2 \mu \mathrm{m}$ keeping the $\mathrm{L}_{\mathrm{SD}}$ constant, while in the second one, only $\mathrm{L}_{\mathrm{GS}}$ is decreased keeping $\mathrm{L}_{\mathrm{GD}}$ constant. The resultant effects on output current and gate-capacitances with varying gate voltage from -15 to 1 $\mathrm{V}$ are analyzed. A drain bias of $15 \mathrm{~V}$ is pre-applied to get the $\mathrm{I}_{\mathrm{D}}-\mathrm{V}_{\mathrm{GS}}$, and $\mathrm{g}_{m}-V_{G S}$ characteristics of the devices. The extracted values of gate-source and gate-drain capacitances $C_{G S}$ and $C_{G D}$ have been used for the physical explanation of the device linearity. From Fig. 4, it can be seen that access region length significantly affects the drain current $I_{D}$ and so the transconductance $\mathrm{g}_{m}$.

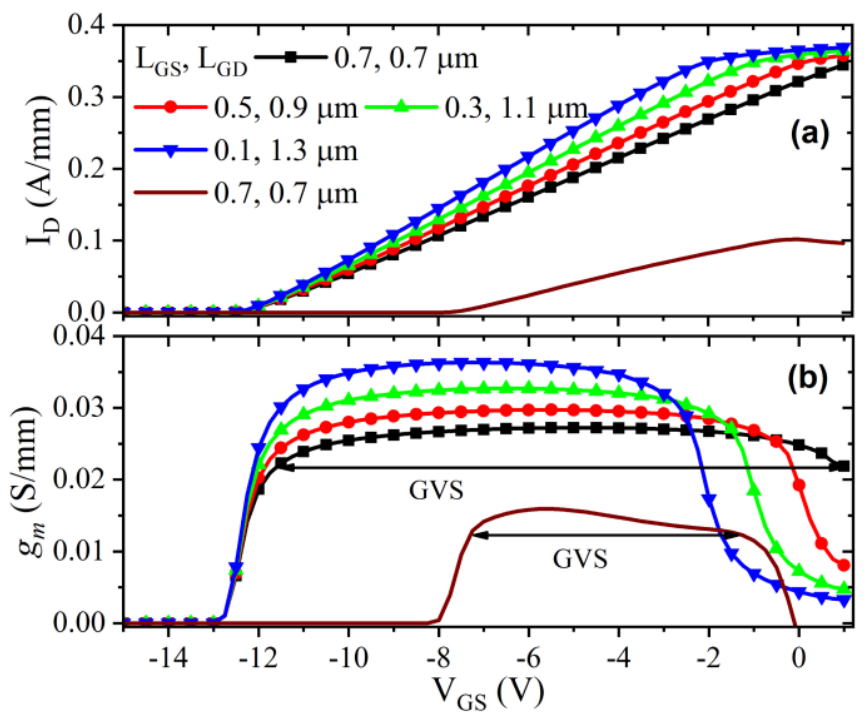

Fig. 4. Effect of varying $L_{G s}$ and $L_{G D}$ on drain current and transconductance of proposed device, $L_{G}=0.1 \mu \mathrm{m}$ for all cases. (a) $I_{D}$ - $\mathrm{V}_{\mathrm{GS}}$ (b) $g_{m}-\mathrm{V}_{\mathrm{GS}}$ characteristics. Line plus symbols are used for proposed device and only line for conventional. Common legends are used. GVS is mentioned to evaluate linearity.

As $L_{G S}$ reduces, lower source-access resistance facilitates higher $\mathrm{I}_{\mathrm{D}}$. Although at higher $V_{G S}$, the drain current starts to saturate. This can be attributed to increased drain-access resistance since $L_{G D}$ keeps increasing. The electric field peak now occurs in the higher resistive drain-access region. This observation is in good agreement with the explanation given in [24]. The linearity of the devices is evaluated based on transconductance curves. A Figure of Merit (FOM) - gate voltage swing (GVS) defined as voltage range for which transconductance does not fall below $20 \%$ of maximum value, is used to measure linearity [13]. For the proposed device, it is measured as greater than $12 \mathrm{~V}$ which is $100 \%$ higher than its respective value of $6 \mathrm{~V}$ for conventional HEMT. The maximum value of transconductance increases as $\mathrm{L}_{\mathrm{GS}}$ decreases, although GVS decreases due to the high resistivity drain-access region as explained earlier and found in good agreement with the phononmodel as explained in [9].

In the second case of analysis, $L_{G S}$ reduces as explained earlier but now $L_{G D}$ is fixed. The appropriate change in $L_{S D}$ is obvious and is incorporated to fit these settings. The resultant effects on $I_{D}$ and $g_{m}$ are shown in Fig. 5. Due to reduced sourceaccess resistance and constant drain-access resistance, here 
drain current $\mathrm{I}_{\mathrm{D}}$ increases proportionally and negligible saturation at higher gate voltage. Consequently, almost flat curves of transconductance $\mathrm{g}_{\mathrm{m}}$ with GVS of $12 \mathrm{~V}$ are measured. These results and observations are endorsed by $\mathrm{C}-\mathrm{V}$ characteristics of the proposed device and are given in the next subsection. The gate-source and gate-drain capacitances $C_{G S}$, and $C_{G D}$ are further validated based on appropriate analytical models.
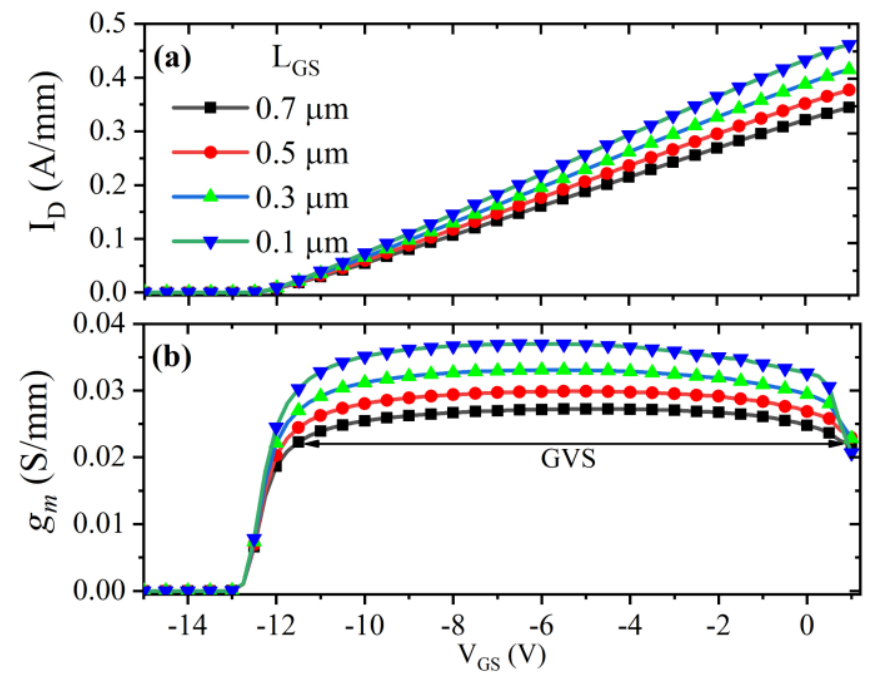

Fig. 5. Effect of varying $L_{G S}$ on drain current and transconductance of proposed device, $\mathrm{L}_{\mathrm{G}}=0.1$ and $\mathrm{L}_{\mathrm{GD}}=0.7 \mu \mathrm{m}$ is fixed. (a) $\mathrm{I}_{\mathrm{D}}-\mathrm{V}_{\mathrm{GS}}$ (b) $g_{m}$ - VGS characteristics. Common legends are used. GVS is almost constant even for higher transconductance value curves.

\section{C - V Analysis}

Since the access region length significantly affects $C-V$ characteristics of the device, small-signal capacitance-voltage analysis is done at a frequency of $1 \mathrm{MHz}$ after applying a drainbias of $15 \mathrm{~V}$. Gate capacitances $C_{G S}$ and $C_{G D}$ are extracted as a function of varying $V_{G S}$ and are shown in Fig. 6 and Fig. 7.

As $V_{G S}$ increases slightly above the cut-off voltage $V_{O F F}, C_{G S}$ values rise vertically and are attributed to the dependence of electron concentration in the channel on $V_{G S}$ as explained in subsection A. It can be seen from Fig. 6 that for all $V_{G S}$ beyond $V_{G S}, C_{G S}$ increases monotonically with decreasing $L_{G S}$, and increasing $L_{G D}$ and plateau is observed. Although a small increase in $C_{G S}$ values are observed at higher $V_{G S}$, corresponding to $L_{G D}=1.1$ and $1.3 \mu \mathrm{m}$, as shown in inset Fig. 6 a. This behavior can be explained on the physics-based compact models as given in [25], [26]. Since the 2DEG density is strong in this region, total gate-capacitance $C_{G}$ is equal to channel capacitance $C_{C H}$ which is defined as $q d n_{s} / d V_{G}$ [25]. It is important to note that due to the doped $\beta-\mathrm{Ga}_{2} \mathrm{O}_{3}$ buffer layer $\left(N_{D}=10^{16} \mathrm{~cm}^{-3}\right)$, these doped charges are also added to $C_{G}$. Now, $C_{G}=C_{C H}+C_{D E P}$, and deletion capacitance is defined as $C_{D E P}=C_{D E P 0} /\left(1+V_{b i} / V_{g, e f f}\right)^{1 / m+2}$ where $V_{b i}$ is the built-in voltage and $C_{D E P}=C_{D E P 0}$ for $V_{g, e f f}>V_{b i}$, and $m$ is the doping profile dependent parameter [25]. Based on this model, it is obvious that as $L_{G D}$ increases more depletion charges are added up to the $C_{G S}$ at higher $V_{G S}$. On the contrary, there is no such divergence in the $C_{G S}-V_{G S}$ characteristics for the second set of analyses is observed, where the $L_{G D}$ is kept constant (Fig.
6 b).

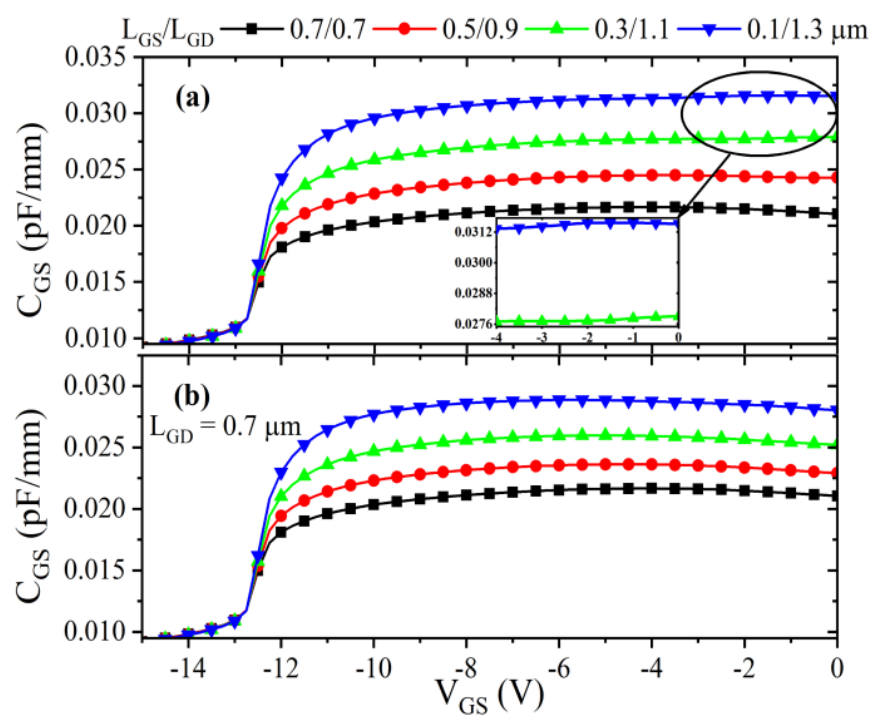

Fig. 6. Effect of changing access lengths on $\mathrm{C}_{\mathrm{GS}}-\mathrm{V}_{\mathrm{GS}}$ characteristics of the proposed device with fixed $L_{G}=0.1 \mu \mathrm{m}$. (a) Both $L_{G S}$ and $L_{G D}$ are changed in the step of $0.2 \mu \mathrm{m}$, Inset: small increase in $\mathrm{C}_{\mathrm{GS}}-\mathrm{V}_{\mathrm{GS}}(-4$ to $0 \mathrm{~V}$ ) relating to $L_{G D}=1.1$ and $1.3 \mu \mathrm{m}$ is highlighted (b) Only $L_{G S}$ is changed and $L_{G D}$ is fixed. Common legends are used.

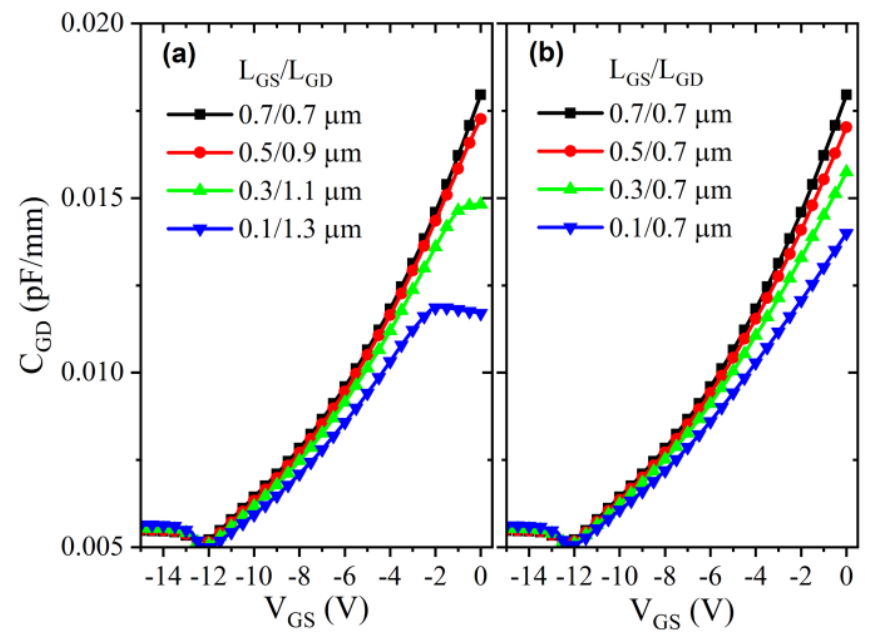

Fig. 7. $C_{G D}-V_{G S}$ characteristics for the proposed device. (a) Both $L_{G S}$ and $L_{G D}$ are varied in tandem. (b) Only $L_{G s}$ is changed, $L_{G D}$ is fixed. In both cases peak value of $C_{G D}$ decreases with $L_{G S}$ scaling, however for $\mathrm{L}_{\mathrm{GD}}=1.1,1.3 \mu \mathrm{m}, \mathrm{C}_{\mathrm{GD}}$ starts to decrease at higher $\mathrm{V}_{\mathrm{GS}}$.

Gate-drain capacitance versus gate voltage for varying access region length is shown in Fig. 7. From both the plots, it can be seen that $C_{G D}$ increases with increasing $V_{G S}$, but the peak value decreases with reducing $L_{G S}$. This can be attributed to the rising values of $C_{G S}$ values with increasing $V_{G S}$ as explained previously based on the analytical model [25] and since total gate-capacitance $C_{G}=C_{G S}+C_{G D}$. Furthermore, for the first case corresponding to $L_{G D}=1.1$ and $1.3 \mu \mathrm{m}, C_{G D}$ starts to decrease beyond $-3 \mathrm{~V}$ of $V_{G S}$, as shown in Fig. 7a. This can be attributed to poor control of the drain electrode as $L_{G D}$ increases. It is validated by invoking the capacitance model [26], and simulations are performed to verify the dependence of $C_{G D}$ on $V_{D S}$ for various values of $V_{G S}$. The plots shown in Fig. 8 are in good agreement with related findings in [26]. 


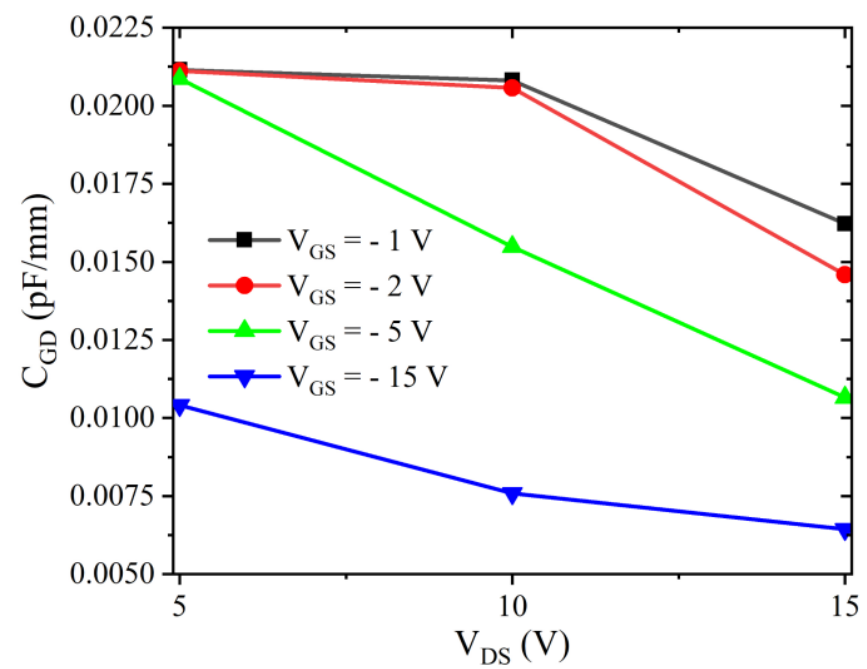

Fig. 8. Effect of higher drain bias on $\mathrm{C}_{\mathrm{GD}}$ at fixed $\mathrm{V}_{\mathrm{GS}}$. Gradual decrease of $\mathrm{C}_{G D}$ with increasing $\mathrm{V}_{D S}$ can be interpreted as steady loss of drain electrode control over channel charge. This is in-line with the capacitance model in [26]

\section{RF Performance}

In order to evaluate the RF performance of the proposed device, a small-signal high-frequency analysis is performed based on the respective simulated transconductance curves. The cut-off frequency $f_{T}$, and associated maximum oscillation frequency $f_{M A X}$ are extracted from the simulated current-gain $h_{21}$ and unilateral power gain $U$ versus frequency characteristics. Fig. 9 shows the simulated values of cut-off frequencies versus gate-voltage. It can be seen that $f_{T}$ dependence on $V_{G S}$ follows a similar trend as transconductance, but with limited linearity. The peak value of $f_{T}$ is found to be $170 \mathrm{GHz}$ which gradually decreases with increasing $V_{G S}$. The fall in $f_{T}$ can be attributed to increasing gate-drain capacitance $C_{G D}$ as shown in Fig. 7. The cut-off frequency $f_{T}$ is also calculated based on the following relation:

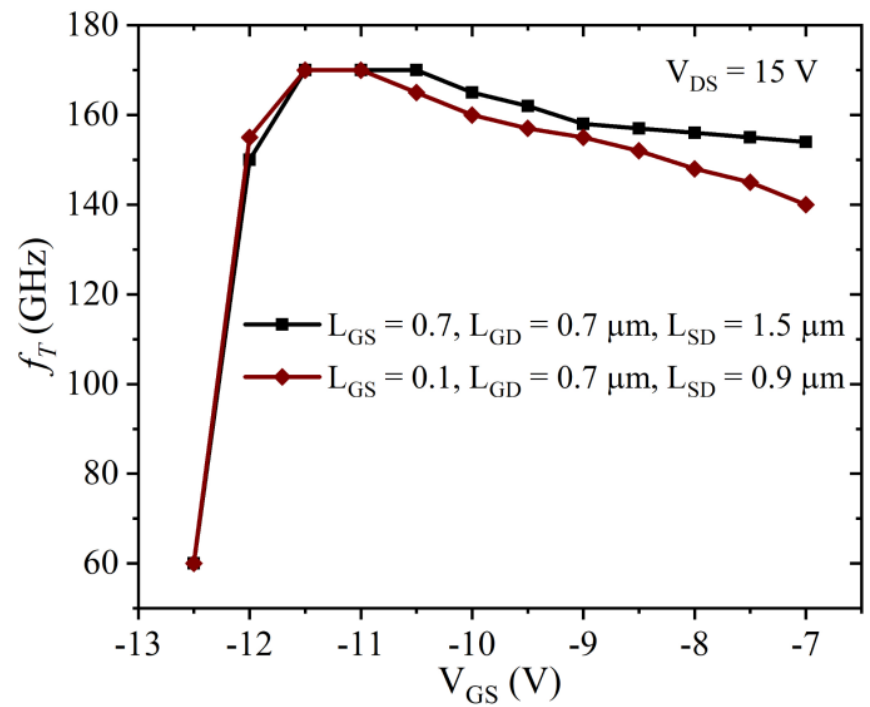

Fig. 9. Plot of extracted cut-off frequency $f_{T}$ versus gate voltage $V_{G S}$ at $V_{D S}=15 \mathrm{~V}$ of the proposed device with gate-length $\mathrm{L}_{\mathrm{G}}=0.1 \mu \mathrm{m}$.

$$
f_{T} \approx \frac{g_{m}}{2 \pi\left(C_{G S}+C_{G D}\right)}
$$

Numerically computed values of $f_{T}$ using (3) are found to be slightly lower than the simulated values, nevertheless follows the similar trend with gate voltage.

\section{CONCLUSION}

The inherent factors limiting the linearity performance of HEMTs are analyzed qualitatively. Simulation results of the proposed HEMT design, which incorporate measures to circumvent these issues, have shown excellent transconductance linearity and moderate cut-off frequency linearity as a function of increasing gate voltage. The effect of varying access region length on the $I_{D}-V_{G S}$ and $g_{m}-V_{G S}$ characteristics are thoroughly investigated. These DC characteristics are explained based on capacitance-voltage measurements which are validated by appropriate physicsbased analytical models. Apart from inherent advantages like simpler design and negligible parasitic capacitances of the planar HEMT, the proposed design equipped with better linearity is expected to be useful in low-noise amplifiers and RF applications.

\section{ACKNOWLEDGMENT}

Visvesvaraya Young Faculty Research Fellowship by Ministry of Electronics and Information Technology (MeitY), Govt. of India being implemented by Digital India Corporation.

\section{REFERENCES}

1. H. He, et al., "First-principles study of the structural, electronic, and optical properties of $\mathrm{Ga}_{2} \mathrm{O}_{3}$ in its monoclinic and hexagonal phases," Physical Review B 74, Art. no. 195123, Nov. 2006, doi: 10.1103/physrevb.74.195123.

2. S. Yoshioka, H. Hayashi, A. Kuwabara, F. Oba, K. Matsunaga, and I. Tanaka, "Structures and energetics of $\mathrm{Ga}_{2} \mathrm{O}_{3}$ polymorphs," J. Phys.: Condens Matter, vol. 19, no. 34, Art. no. 346211, Jul. 2007, doi: 10.1088/0953-8984/19/34/346211.

3. M. Higashiwaki, K. Sasaki, A. Kuramata, T. Masui, and S. Yamakoshi, "Gallium oxide $\left(\mathrm{Ga}_{2} \mathrm{O}_{3}\right)$ metal semiconductor field effect transistors on single crystal $\beta-\mathrm{Ga}_{2} \mathrm{O}_{3}(010)$ substrates," Appl. Phys. Lett., vol. 100, Art. no. 013504, Jan. 2012, doi: 10.1063/1.3674287.

4. S. Poncé and F. Giustino, "Structural, electronic, elastic, power, and transport properties of $\beta-\mathrm{Ga}_{2} \mathrm{O}_{3}$ from first principles" Phys. Rev. Research 2, Art. no. 033102, Jul. 2020, doi: 10.1103/PhysRevResearch.2.033102

5. S. J. Pearton, F. Ren, M. Tadjer, and J. Kim, "Perspective: $\mathrm{Ga}_{2} \mathrm{O}_{3}$ for ultrahigh power rectifiers and MOSFETS," J. Appl. Phys. 124, Art no. 220901, Dec. 2018, doi: 10.1063/1.5062841.

6. E. Ahmadi, and Y. Oshima, "Materials issues and devices of $\alpha$ - and $\beta-$ $\mathrm{Ga}_{2} \mathrm{O}_{3}$," J. Appl. Phys. 126, Art. no. 160901, Oct. 2019, doi: 10.1063/1.5123213.

7. R. Singh et al., "The Dawn of $\mathrm{Ga}_{2} \mathrm{O}_{3}$ HEMTs for High Power Electronics - A Review," Materials Science in Semiconductor Processing , vol. 119, no. 105216, May 2020, doi:10.1016/j.mssp.2020.105216.

8. T. Palacios, S. Rajan, A. Chakraborty, S. Heikman, S. Keller, S.P. DenBaars, U.K. Mishra, "Influence of the Dynamic Access Resistance in the gm and $\mathrm{f}_{\mathrm{T}}$ Linearity of $\mathrm{AlGaN} / \mathrm{GaN}$ HEMTs" IEEE Trans. Electron Devices, vol. 52, no. 10, pp. 2117-2123, Oct. 2005, doi: 10.1109/TED.2005.856180

9. T. Fang, R. Wang, H. Xing, S. Rajan, and D. Jena, "Effect of Optical Phonon Scattering on the Performance of GaN Transistors" IEEE Electron Device Lett., vol. 33, no. 5, pp. 709-711, May 2012, doi: 10.1109/LED.2012.2187169 
10. J. Chang et al., "The super-lattice castellated field-effect transistor: A high-power, high-performance RF amplifier," IEEE Electron Device Lett., vol. 40, no. 7, pp. 1048-1051, Jul. 2019, doi: 10.1109/LED.2019.2917285.

11. P. Shrestha et al., "High linearity and high gain performance of $\mathrm{N}$-polar GaN MIS-HEMT at $30 \mathrm{GHz}$," IEEE Electron Device Lett., vol. 41, no. 5, pp. 681-684, May 2020, doi: 10.1109/LED.2020.2980841.

12. S. Bajaj et al., "Graded AlGaN channel transistors for improved current and power gain linearity," IEEE Trans. Electron Devices, vol. 64, no. 8, pp. 3114-3119, Aug. 2017, doi: 10.1109/TED.2017.2713784.

13. O. Odabasi et al., "AlGaN/GaN-Based Laterally Gated High-ElectronMobility Transistors With Optimized Linearity," IEEE Trans. Electron Devices, vol. 68, no. 3, Mar. 2021, doi: 10.1109/TED.2021.3053221.

14. S. Ghosh, S. A. Ahsan, Y. S. Chauhan and S. Khandelwal, "Modeling of source/drain access resistances and their temperature dependence in $\mathrm{GaN}$ HEMTs," 2016 IEEE International Conference on Electron Devices and Solid-State Circuits (EDSSC), 2016, pp. 247-250, doi: 10.1109/EDSSC.2016.7785254.

15. I. G. Lee, et al., "Theoretical and experimental analysis of the source resistance components in $\mathrm{In}_{0.7} \mathrm{Ga}_{0.3}$ As quantum-well high-electronmobility transistors." Journal of the Korean Physical Society 78, no. 6, pp. 516-522, Feb. 2021, doi: 10.1007/s40042-021-00096-0.

16. P. Cui et al., "Influence of Different Gate Biases and Gate Lengths on Parasitic Source Access Resistance in AlGaN/GaN Heterostructure FETs," IEEE Trans. Electron Devices, vol. 64, no. 3, pp. 1038-1044, Mar. 2017, doi: 10.1109/TED.2017.2654262.

17. R. Singh, T. R. Lenka, and H. P. T. Nguyen, "Optimization of Dynamic Source Resistance in a $\beta-\mathrm{Ga}_{2} \mathrm{O}_{3}$ HEMT and Its Effect on Electrical Characteristics," Journal of Elec. Materi. vol 49, no. 9, pp. 5266-5271, Jun. 2020, doi: 10.1007/s11664-020-08261-0.

18. Z. Xia et al., " $\beta-\mathrm{Ga}_{2} \mathrm{O}_{3}$ Delta-Doped Field-Effect Transistors With Current Gain Cutoff Frequency Of $27 \mathrm{GHz}$," IEEE Electron Device Lett., vol. 40, no. 7, pp. 1052-1055, Jul. 2019, doi: 10.1109/LED.2019.2920366

19. Device Simulation Software, ATLAS User's Manual, Silvaco, Santa Clara, CA, USA, 2009.

20. MATLAB. version 7.10.0 (R2018a). Natick, Massachusetts: The MathWorks Inc.; 2018.

21. K. Ghosh and U. Singisetti, "Ab initio velocity-field curves in monoclinic $\beta-\mathrm{Ga}_{2} \mathrm{O}_{3}$ ” J. Appl. Phys. vol. 122, no. 3, 035702 (1-7), Jul. 2017, doi: 10.1063/1.4986174.

22. S. Kumar, R. Soman, A. S. Pratiyush, R. Muralidharan, and D. N. Nath, "A Performance Comparison Between $\beta-\mathrm{Ga}_{2} \mathrm{O}_{3}$ and GaN HEMTs." IEEE Trans. Electron Devices, vol. 66, no. 8, pp. 3310- 3317, (2019).

23. S. Khandelwal, N. Goyal, and T. A. Fjeldly, "A Physics-Based Analytical Model for 2DEG Charge Density in AlGaN/GaN HEMT Devices," IEEE Trans. Electron Devices, vol. 58, no. 10, pp. 3625-2123, Oct. 2011, doi: 10.1109/TED.2011.2161314.

24. K. Sharma, A. Dasgupta, S. Ghosh, S. A. Ahsan, S. Khandelwal, and Y. S. Chauhan, "Effect of Access Region and Field Plate on Capacitance behavior of GaN HEMT" IEEE International Conference on Electron Devices and Solid-State Circuits (EDSSC), pp. 499-502, Jun 2015, doi:10.1109/edssc.2015.7285160.

25. S. Khandelwal and T. A. Fjeldly, "A Physics Based Compact Model of Gate Capacitance in AlGaN/GaN HEMT Devices," 8th International Caribbean Conference on Devices, Circuts and Systems (ICCDCS), pp. 14, Mar. 2012, doi:10.1109/iccdes.2012.6188891.

26. S. Khandelwal and T. A. Fjeldly, "A physics based compact model of I$\mathrm{V}$ and $\mathrm{C}-\mathrm{V}$ characteristics in AlGaN/GaN HEMT devices," Solid-State Electronics, 76, pp.60-66, Oct. 2012, doi: 10.1016/j.sse.2012.05.054. 